\title{
Parameter Identification for Ultrasound Shear Wave Elastography Simulation
}

\begin{abstract}
Elasticity of soft tissue is a valuable information to physicians in treatment and diagnosis of diseases. The elastic properties of tissue can be estimated with ultrasound (US) shear wave imaging (SWEI). In US-SWEI, a force push is applied inside the tissue and the resulting shear wave is detected by high-frequency imaging. The properties of the wave such as the shear wave velocity can be mapped to tissue elasticity. Commonly, wave features are extracted by tracking the peak of the shear wave, estimating the phase velocity or with machine learning methods. To tune and test these methods, often simulation data is employed since material properties and excitation can be accurately controlled. Subsequent validation on real US-SWEI data is in many cases performed on tissue phantoms such as gelatine. Clearly, validation performance of these procedures is dependent on the accuracy of the simulated tissue phantom and a thorough comparison of simulation and experimental data is needed. In this work, we estimate wave parameters from 400 US-SWEI data sets acquired in various homogeneous gelatine phantoms. We tune a linear material model to these parameters. We report an absolute percentage error for the shear wave velocity between simulation and phantom experiment of $<2.5 \%$. We validate our material model on unknown gelatine concentrations and estimate the shear wave velocity with an error $<3.4 \%$ for in-range concentrations indicating that our material model is in good agreement with US-SWEI measurements
\end{abstract}

Keywords: Ultrasound, Shear Wave Elastography, Shear Wave Simulation, High-Frequency US Imaging, Abaqus

https://doi.org/10.1515/cdbme-2021-1008

\footnotetext{
M Neidhardt' ${ }^{1}$, A. Schlaefer

Institute of Medical Technology and Intelligent Systems, Hamburg University of Technology, Hamburg, Germany

e-mail: Maximilian.Neidhardt@tuhh.de

\section{J. Ohlsen ${ }^{1}$, N. Hoffmann}

The Dynamics Group, Hamburg University of Technology, Hamburg, Germany

${ }^{1}$ Both authors contributed equally.
}

\section{Problem}

Pathological properties of tissue change due to diseases. Hence, quantitative estimates of the elastic shear stiffness of tissue have been proven as a valuable marker in disease diagnostic and staging [4]. To estimate elastic shear stiffness of tissue we use ultrasound (US) shear wave elastography imaging (SWEI). A disturbance is induced inside the tissue by performing an acoustic radiation force push. As a result, a shear wave propagates through the tissue with its wave characteristics, such as the velocity, dependent on the tissue properties.

Simulations are a common tool in US-SWEI for tuning image processing methods or generating large datasets for machine learning methods. Commonly, gelatine phantoms are modelled in simulations since they allow an experimental validation and are less complex to control. Gelatine is a viscohyperelastic material and the choice of a suitable material model for numerical simulations and the determination of the respective material parameters is not trivial and often depends on the specific case of application. A common way to determine material parameters of tissue-like materials is by performing compression [6] or indentation tests [1].

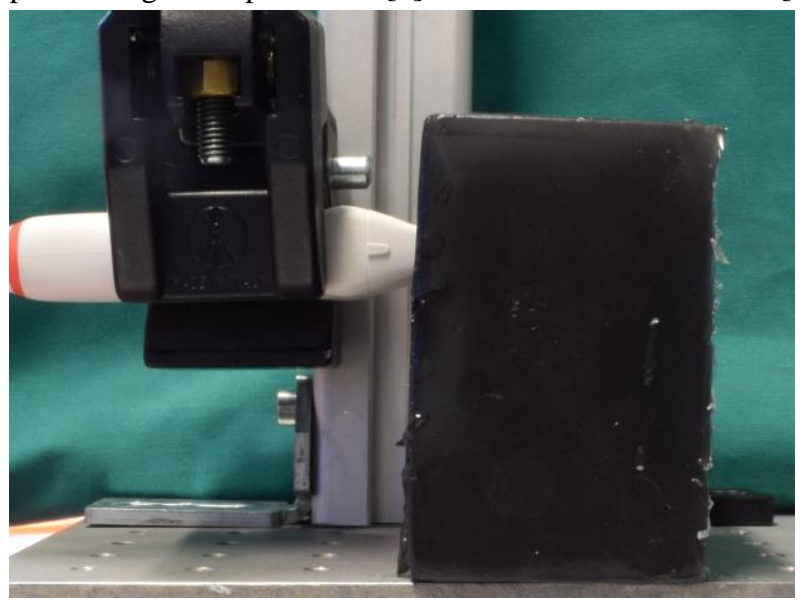

Figure 1: Experimental setup with a linear array ultrasound transducer and a homogeneous gelatine phantom. We acquire shear wave data on six different gelatine concentrations ranging from $2.5 \%$ to $15.0 \%$. On each phantom we perform 80 pushes at various locations on the surface. 

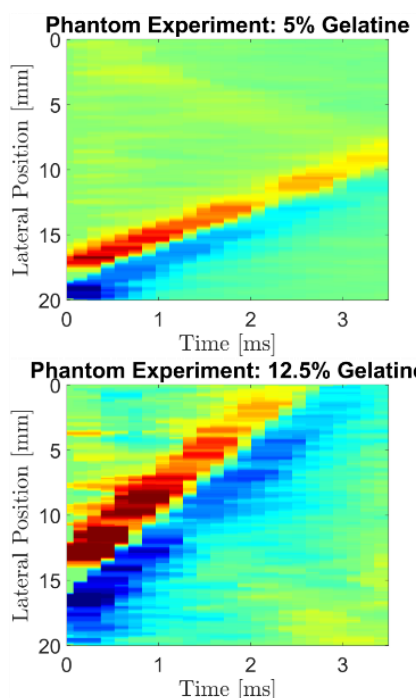
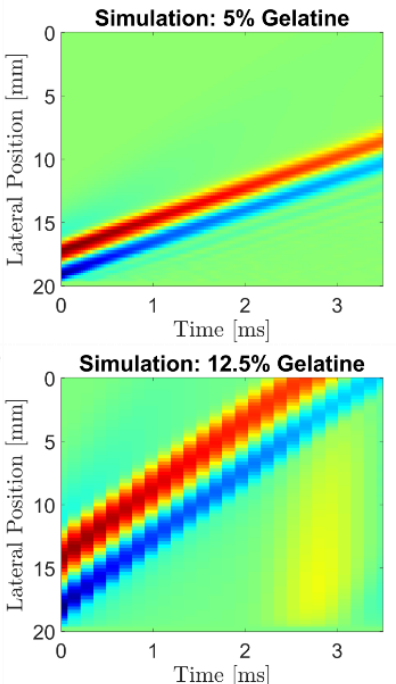

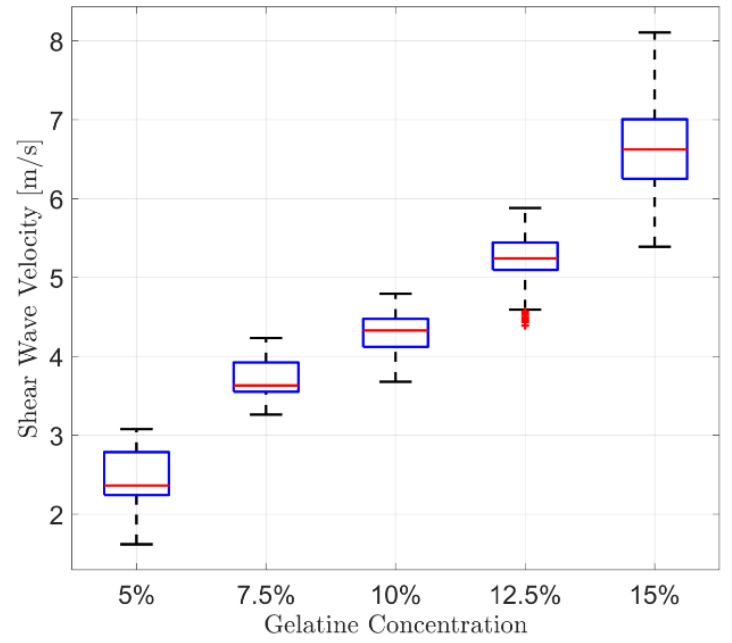

Figure 2: Left: The shear wave velocity and wavelength were estimated from spatio-temporal data by linear regression. Please note that only one wave from the center push to the side is visible. The red and blue indicate the normalized crests and troughs of the shear wave. In the left column is the pre-processed US shear wave data for $5 \%$ and $12.5 \%$ gelatine concentration. In the right column is the simulated shear wave data on identical concentrations. Right: Shear wave velocities estimated from US-SWEl in homogeneous gelatin phantoms. Each box plot corresponds to 80 measurement

However, displacements induced through a shear wave are below $100 \mu \mathrm{m}$ hence an indentation experiment is challenging in this range. In this work, we measure wave characteristics for different gelatine concentrations directly from US-SWEI data. We present an experimental setup to acquire a large dataset of propagating shear waves in phantoms with various gelatine concentrations. We tune a linear elastic material model and validate simulated displacement fields on unknown gelatin concentrations.

\section{Material and Methods}

To evaluate our wave field simulation, we acquire US shear wave data of homogeneous gelatine phantoms. We tune the parameters of the material model and simulation based on wavelength and velocity extracted from the acquired USSWEI data.

\subsection{Experimental Setup}

The setup for shear wave US data acquisition is depicted in Fig. 1. We manufacture homogeneous ballistic gelatine phantoms (GELITA ballistic type 3) with a gelatine to water concentration of $5 \%, 7.5 \%, 10 \%, 12.5 \%$ and $15 \%$ with a rectangular block size of $100 \mathrm{~mm} \times 100 \mathrm{~mm} \times 70 \mathrm{~mm}$. For shear wave imaging we employ a high-speed US imaging device
(Cephasonics, Cicada) with a $7.5 \mathrm{MHz}$ linear array transducer. We generate US pushes at a depth of $10 \mathrm{~mm}$. We track the resulting shear wave with plane wave imaging at 7000 frames per second and record raw in-phase and quadrature (IQ) US signals. The resolution of the US images after offline beam forming is 250pixels $\times 600$ pixels or $20 \mathrm{~mm} \times 33.8 \mathrm{~mm}$ along the depth and lateral axis, respectively. We acquire 30 images after each push sequence. For each gelatine concentration 80 US-SWEI measurements are performed, yielding a total of 400 measurements.

\subsection{Extracting Parameters}

To estimate particle motion between two consecutive time frames we apply the Loupas' autocorrelator algorithm [5] on the IQ signals. Further, we reduce reflection artifacts and noise with a 2-dimensional directional filter in the frequency domain [3]. To enhance the signal-to-noise ratio we apply a 3dimensional median filter with a kernel size of $5 \mathrm{px}$ in each direction. We estimate the shear wave velocity by extracting $1 \mathrm{D}$ signals along the lateral direction at the depth of the applied push. We stack the extracted 1D signals of consecutive images results in a 2 -dimensional image of size $600 \times 30$ along the lateral and time axis, respectively. 
Table 1: Results from the phantom experiments with ultrasound shear wave imaging and simulation. Given is the shear wave velocity $c$, shear modulus $\mu$ and wave length $\lambda$. The shear modulus $\mu$ is set as material model parameter in the simulation. The error between extracted parameters of the simulation and phantom experiment is given as the absolute percentage error (APE).

\begin{tabular}{|c|c|c|c|c|c|c|c|c|}
\hline \multirow[b]{2}{*}{ Gelatine [\%] } & \multicolumn{3}{|c|}{ Phantom Experiments } & \multicolumn{5}{|c|}{ Simulation } \\
\hline & $\mu_{p}[\mathrm{kPa}]$ & $\mathrm{c}_{p}[\mathrm{~m} / \mathrm{s}]$ & $\lambda_{p}[\mathrm{~mm}]$ & $\mu_{\text {sim }}[\mathrm{kPa}]$ & $\mathrm{c}_{\operatorname{sim}}[\mathrm{m} / \mathrm{s}]$ & $\operatorname{APE}_{c}[\%]$ & $\lambda_{\text {sim }}[\mathrm{mm}]$ & $\mathrm{APE}_{\lambda}[\%]$ \\
\hline 5 & $5.93 \pm 2.02 a$ & $2.41 \pm 0.38$ & $3.84 \pm 0.32$ & 5.93 & $2.36 \pm 0.01$ & 2.11 & $3.39 \pm 0.03$ & 11.72 \\
\hline 7.5 & $14.20 \pm 1.98$ & $3.71 \pm 0.25$ & $5.28 \pm 0.58$ & 14.20 & $3.37 \pm 0.01$ & 0.59 & $5.46 \pm 0.02$ & 3.41 \\
\hline 10 & $19.29 \pm 2.59$ & $4.30 \pm 0.28$ & $5.78 \pm 0.44$ & 19.29 & $4.24 \pm 0.01$ & 1.28 & $6.19 \pm 0.18$ & 7.10 \\
\hline 12.5 & $28.97 \pm 3.30$ & $5.24 \pm 0.29$ & $6.78 \pm 0.66$ & 28.97 & $5.23 \pm 0.01$ & 0.22 & $7.35 \pm 0.05$ & 8.41 \\
\hline 15 & $46.75 \pm 8.40$ & $6.62 \pm 0.57$ & $9.84 \pm 1.48$ & 46.75 & $6.68 \pm 0.01$ & 0.92 & $9.46 \pm 0.05$ & 4.35 \\
\hline
\end{tabular}

Table 2: Results for fitting the shear modulus to a third order polynomial and estimating the shear wave velocity $\mathrm{C}_{\text {simlnt }}$ and wavelength $\lambda_{\text {simlnt }}$. The APE is given as the error between phantom experiments and simulation.

\begin{tabular}{|c|c|c|c|c|c|c|}
\hline Gelatine [\%] & $\mu_{\text {simlnt }}[\mathrm{kPa}]$ & $\mathrm{APE}_{\mu} \mathrm{Int}[\%]$ & $\mathrm{c}_{\operatorname{sim} / n t}[\mathrm{~m} / \mathrm{s}]$ & $\mathrm{APE}_{c / n t}[\%]$ & $\lambda_{\text {simlnt }}[\mathrm{mm}]$ & $\mathrm{APE}_{\lambda l n t}[\%]$ \\
\hline 5 & 10.18 & 71.80 & $3.31 \pm 0.01$ & 30.25 & $4.51 \pm 0.08$ & 17.14 \\
\hline 7.5 & 13.14 & 7.49 & $3.58 \pm 0.01$ & 3.36 & $5.24 \pm 0.03$ & 0.76 \\
\hline 10 & 20.00 & 3.68 & $4.32 \pm 0.01$ & 0.53 & $6.30 \pm 0.20$ & 9.00 \\
\hline 12.5 & 27.90 & 3.67 & $5.10 \pm 0.02$ & 2.62 & $7.11 \pm 0.04$ & 4.87 \\
\hline 15 & 51.00 & 9.10 & $7.02 \pm 0.02$ & 6.08 & $9.90 \pm 0.09$ & 0.10 \\
\hline
\end{tabular}

Example images are given on the left in Fig.2. We detect the crests and troughs of the wave by finding the position of the minimum and maximum along the lateral axis for each time instance. To retrieve the steepness of the curve we perform a linear regression for the crests and troughs points in both directions. We estimate four linear regression models for each push and imaging sequence in total. The steepness of the linearly fitted curve is directly related to the shear wave velocity. Similar, we can estimate the wavelength by the lateral distance between the crests and troughs at each time instance.

\subsection{Simulation Environment}

We employ the numerical software Abaqus/CAE 2018 [8] to perform two dimensional simulations mimicking the events in the imaging plane of the experiments described in Section 2.1. A rectangular geometry is discretized using 25312 triangular mesh elements (Abaqus Type CPS6M) and excited by a sinusoidal displacement of a mesh node at its midpoint. To conform with the ultrasound push of the experimental setup, the excitation is limited to the first cycle of the sinusoidal displacement. The mean excitation frequency $f$ was derived from the measured wavelengths $\lambda$ and velocities $\mathrm{c}$ with $\mathrm{f}=\mathrm{c} / \lambda$.
The numerical experiments are performed assuming an elastic, isotropic and quasi-incompressible material behaviour [2]. For each gelatine concentration we derive the shear modulus $\mu$ from the mean shear wave velocity $\mathrm{c}$ which was estimated in the phantom experiment. It is computed by $\mu=c^{2} \rho$ with $\rho$ as the density [7]. The Young's modulus E can be gained with $\mathrm{E}=2 \mu^{2}(1+v)$ where the Poisson's ratio $v$ is chosen as 0.4999 to prevent numerical instabilities.

In a post-processing step, the simulated displacement fields are interpolated on a Cartesian grid of $250 \times 600 \times 30$ along the depth-, lateral- and time axis, respectively. This aligns the spatio-temporal coordinates of the simulated data with the US-SWEI phantom data and thereby allows identical data processing pipelines for both data sources.

To validate our simulation, we exclude a single gelatine concentration and tune the simulation parameters on the remaining experimental data sets. We estimate the shear modulus of the excluded gelatine concentration with a third order polynomial regression approach. After post-processing the simulation data, we estimate the shear wave velocity and wavelength as described in Sec. 2.2. 

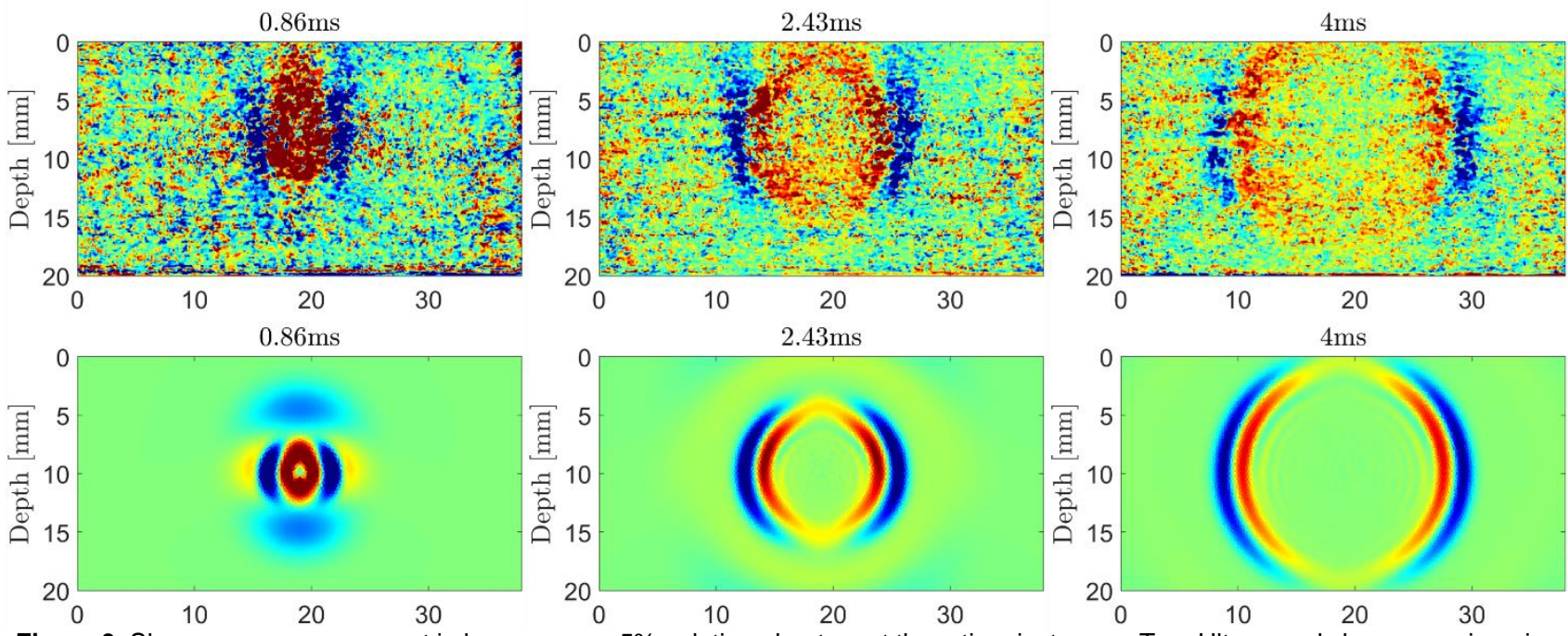

Figure 3: Shear wave measurement in homogenous $5 \%$ gelatine phantom at three time instances. Top: Ultrasound shear wave imaging.

\section{Results}

All simulations were performed with an excitation frequency of $704 \mathrm{~Hz}$. Fig. 2 shows that we can distinguish the gelatine concentrations based on the shear wave velocity estimated from US-SWEI phantom data. Estimates of the Young's Modulus and wave length from our phantom experiments are given in Table 1, which yields a decreasing shear wave velocity for softer phantoms with a lower gelatine concentration. The wave length increases for phantoms with a higher gelatine concentration. The mean of the absolute percentage error (APE) for the shear wave velocity between simulation and phantom experiment is $4.25 \%$ and for the Bottom: simulation of wave field with Abaqus.

\section{Author Statement}

Research funding: This work was partially funded by the TUHH i ${ }^{3}$ initiative and partially by DFG SCHL 1844/2-2.

Conflict of interest: Authors state no conflict of interest. Informed consent: Informed consent has been obtained from all individuals included in this study.

Ethical approval: No ethical approval was necessary for this research.

\section{References}

[1] Abdolazizi, K.P., Linka, K., Sprenger, J., Neidhardt, M., Schlaefer, A., Cyron, C.J.: Concentration-specific constitutive modeling of gelatin based on artificial neural networks. PAMM 20(1), e202000,284 (2021)

[2] Ahmed, H., Salem, N., Seddik, A., Eladawy, M.: On shear wave speed estimation for agar-gelatine phantom. IJACSA 7 (2016). 10.14569/IJACSA.2016.070255

[3] Deffieux, T., Gennisson, J.L., Bercoff, J., Tanter, M.: On the effects of reflected waves in transient shear wave elastography. IEEE UFFC 58(10), 2032-2035 (2011)

[4] Ferraioli, G., Parekh, P., Levitov, A.B., Filice, C.: Shear wave elastography for evaluation of liver fibrosis. J Ultrasound Med 33(2), 197-203 (2014)

[5] Loupas, T., Powers, J., Gill, R.W.: An axial velocity estimator for ultrasound blood flow imaging, based on a full evaluation of the doppler equation by means of a two-dimensional autocorrelation approach. IEEE UFFC 42(4), 672-688 (1995)

[6] Ravikumar, N., Noble, C., Cramphorn, E., Taylor, Z.A.: A constitutive model for ballistic gelatin at surgical strain rates. $J$ Mech Behav Biomed Mater 47, 87-94 (2015)

[7] Sarvazyan, A.P., Rudenko, O.V., Swanson, S.D., Fowlkes, J., Emelianov, S.Y.: Shear wave elasticity imaging: a new ultrasonic technology of medical diagnostics. Ultrasound Med. Biol. 24(9), 1419-1435 (1998)

[8] Smith, M.: ABAQUS User's Manual, Version 2018. Dassault Systèmes Simulia Corp, United States (2018) 